\title{
7-羟乙基白杨素对低压性缺氧大鼠运动性疲劳 具有保护作用
}

苗路伟 ${ }^{1,2}$, 赵 䀐 $^{1,2}$, 高迎春 ${ }^{2}$, 景临林 ${ }^{2}$, 黄 琼 $^{3}$, 马慧萍 1,2

1. 甘肃中医药大学药学院, 甘肃兰州 730000

2. 中国人民解放军联勤保障部队第九四○医院药剂科 全军高原医学实验室, 甘肃 兰州 730050

3. 中国人民解放军联勤保障部队第九四 ○医院医疗保障中心, 甘肃兰州 730050

[摘 要] 目的:探究 7-差乙基白杨素 (7-HEC)对低压性缺氧大鼠运动性疲劳的保 护作用。方法: 选取 40 只健康雄性Wistar 大鼠随机分为常氧对照组、缺氧对照组、 白杨素组、7-HEC 组, 每组各 10 只, 白杨素组和 7-HEC 组分别给予白杨素和 7-HEC $350 \mathrm{mg} / \mathrm{kg}$, 常氧对照组和缺氧对照组给予等量灭菌注射用水。常氧对照组饲养于 当地海拔; 其余三组置于低压性缺氧动物实验舱模拟 $7000 \mathrm{~m}$ 海拔进行缺氧处理。连 续灌胃给药 $3 \mathrm{~d}$ 后进行力竭实验, 统计各组游泳时间。力竭实验后立即采集大鼠的 血液、肝组织和骨骼肌组织, 观察肝组织和骨骼肌组织病理学变化, 测定血尿素 氮、乳酸、丙二醛、总超氧化物歧化酶(T-SOD)、肌肝糖原含量等指标。结果: 与 缺氧对照组比较, 7-HEC 组负重游泳时间延长 [(2.58 0 0.77)和 $(4.04 \pm 1.30) \mathrm{min}$, $P<0.01]$; 肝组织和骨骼肌组织的病理学变化改善; 血尿素氮 [ (3.24 \pm 0.93$)$ 和 $\left.(2.22 \pm 0.60) \mathrm{mmol} \cdot \mathrm{L}^{-1} \cdot \mathrm{min}^{-1}\right]$ 、乳酸 $[$ 肝组织为 $(0.14 \pm 0.05)$ 和 $(0.10 \pm$ $0.03) \mathrm{mg} \cdot \mathrm{g}^{-1} \cdot \mathrm{min}^{-1}$, 骨骼肌组织为 $(0.26 \pm 0.06)$ 和 $\left.(0.18 \pm 0.07) \mathrm{mg} \cdot \mathrm{g}^{-1} \cdot \mathrm{min}^{-1}\right]$ 、 丙二醛 $\left[\right.$ 肝组织为 $(1.37 \pm 0.48)$ 和 $(0.78 \pm 0.28) \mathrm{nmol} \cdot \mathrm{mg}^{-1} \cdot \mathrm{min}^{-1}$, 骨骼肌组织为 $(0.87 \pm 0.19)$ 和 $\left.(0.63 \pm 0.11) \mathrm{nmol} \cdot \mathrm{mg}^{-1} \cdot \mathrm{min}^{-1}\right]$ 生成速率减小 $($ 均 $P<0.05)$; 糖原 [肝组织为 $(15.16 \pm 2.69)$ 和 $(18.89 \pm 2.88) \mathrm{mg} / \mathrm{g}$, 骨骼肌组织为 $(1.46 \pm 0.49)$ 和 $(1.78 \pm 0.48) \mathrm{mg} / \mathrm{g}$ ] 和 T-SOD [ 肝组织为 $(1.87 \pm 0.01)$ 和 $(2.68 \pm 0.12) \mathrm{U} / \mathrm{mL}$, 骨骼 肌组织为 $(5.46 \pm 0.42)$ 和 $(6.52 \pm 0.96) \mathrm{U} / \mathrm{mL}]$ 增加 (均 $P<0.05)$ 。结论: 7-HEC 对 低压性缺氧大鼠运动性疲劳具有明显保护作用。

[关键词 ] 7-差乙基白杨素;抗疲劳;低压性缺氧; 力竭实验; 大鼠

[中图分类号 ］R965.1［文献标志码］A

\section{收稿日期: 2021-02-05 接受日期:2021-07-25 网络预发表日期: 2021-10-21}

基金项目: 国家自然科学基金 (81571847,81872796); 军队后勤科研计划(CWH17J010,CLB18J028)

第一作者:苗路伟, 硕士研究生, 主要从事抗高原缺氧新药研究;E-mail :3045564500@qq.com; https ://orcid.org/0000-00029961-1842.

通信作者:马慧萍,主任药师,教授, 硕士生导师,主要从事高原缺氧损伤机制及新药研发工作;E-mail: 1026573411@qq.com; https ://orcid.org/0000-0003-4560-2155. 黄 琼,主任护师,主要从事医疗保障工作;E-mail:mahuiping2020@163.com; https ://orcid.org/0000-0002-0335-9049. 


\section{Protective effects of 7-hydroxyethyl chrysin on rats with exercise-induced fatigue in hypobaric hypoxia environment} MIAO Luwei ${ }^{1,2}$, ZHAO Tong ${ }^{1,2}$, GAO Yingchun ${ }^{2}$, JING Linlin ${ }^{2}$, HUANG Qiong ${ }^{3}$, MA Huiping $^{1,2}$ (1. College of Pharmacy, Gansu University of Traditional Chinese Medicine, Lanzhou 730000, China; 2. Key Laboratory of the Plateau Medicine, the 940th Hospital of Joint Logistics Support Force of Chinese People's Liberation Army, Lanzhou 730050, China; 3. Medical Support Center, the 940th Hospital of Joint Logistics Support Force of Chinese People's Liberation Army, Lanzhou 730050, China)

Corresponding authors: MA Huiping, E-mail: 1026573411@qq.com, https://orcid.org/ 0000-0003-4560-2155; HUANG Qiong, E-mail: mahuiping2020@163.com, https://orcid. org/0000-0002-0335-9049.

[ Abstract ] Objective: To investigate the protective effect of 7-hydroxyethyl chrysin (7HEC) on rats with exercise-induced fatigue in hypobaric hypoxic condition. Methods: Forty healthy male Wistar rats were randomly divided into four groups with 10 rats in each group: control group, model group, chrysin group and 7-HEC group. The rats in control group were raised at local altitude but other three groups were raised in a simulating altitude of $7000 \mathrm{~m}$ for hypobaric hypoxia treatment. The chrysin group and 7-HEC group were given $350 \mathrm{mg} / \mathrm{kg}$ chrysin or 7-HEC by gavage for $3 \mathrm{~d}$, respectively; while the control group and model group were given the same amount of sterilized water. The weight-bearing swimming tests were performed $3 \mathrm{~d}$ later, and the weight-bearing swimming time was documented. After rats were sacrificed, the liver and skeletal muscle tissue samples were taken for pathological examination and determination of lactate, malondialdehyde (MDA), total superoxide dismutase (T-SOD) and glycogen levels. Blood urea nitrogen was also determined. Results: Compared with the model group, weight-bearing swimming times were significantly prolonged in 7-HEC group $[(2.58 \pm 0.77)$ vs. $(4.04 \pm 1.30) \mathrm{min}, P<0.01]$; pathological changes in liver and skeletal muscle tissue were attenuated; generation rate of blood urea nitrogen $\left[(3.24 \pm 0.93)\right.$ vs. $\left.(2.22 \pm 0.60) \mathrm{mmol} \cdot \mathrm{L}^{-1} \cdot \min ^{-1}, P<0.05\right]$, lactate [liver: $(0.14 \pm 0.05)$ vs. $(0.10 \pm 0.03) \mathrm{mg} \cdot \mathrm{g}^{-1} \cdot \mathrm{min}^{-1}$, skeletal muscle: $(0.26 \pm 0.06)$ vs. $\left.(0.18 \pm 0.07) \mathrm{mg} \cdot \mathrm{g}^{-1} \cdot \mathrm{min}^{-1}\right]$ and MDA [liver: $(1.37 \pm 0.48)$ vs. $(0.78 \pm 0.28) \mathrm{nmol} \cdot \mathrm{mg}^{-1} \cdot \mathrm{min}^{-1}$, skeletal muscle: $(0.87 \pm 0.19)$ vs. $\left.(0.63 \pm 0.11) \mathrm{nmol} \cdot \mathrm{mg}^{-1} \cdot \mathrm{min}^{-1}\right]$ were significantly reduced (all $P<$ $0.05)$; glycogen content [liver: $(15.16 \pm 2.69)$ vs. $(18.89 \pm 2.88) \mathrm{mg} / \mathrm{g}$, skeletal muscle: $(1.46 \pm 0.49)$ vs. $(1.78 \pm 0.48) \mathrm{mg} / \mathrm{g}$ ] and T-SOD [liver: $(1.87 \pm 0.01)$ vs. $(2.68 \pm 0.12) \mathrm{U} / \mathrm{mL}$, skeletal muscle: $(5.46 \pm 0.42)$ vs. $(6.52 \pm 0.96) \mathrm{U} / \mathrm{mL}]$ were significantly improved (all $P<0.05)$. Conclusion: 7-HEC has significant protective effect on the rats with exerciseinduced fatigue in hypobaric hypoxia condition.

[ Key words ] 7-hydroxyethylchrysin; Anti-fatigue; Hypobaric hypoxia; Exhaustive exercise; Rats

[J Zhejiang Univ (Med Sci), 2021, 50(5): 575-581.]

[缩略语］ 7-差乙基白杨素(7-hydroxyethyl chrysin,7-HEC); 总超氧化物歧化酶(total superoxide dismutase,T-SOD); 苏木精一伊红染色 (hematoxylin and eosin staining, HE 染色) 
疲劳是一种复杂的生理病理现象, 过度瘦劳 会造成人体内分泌紊乱, 免疫力下降, 工作效率低 下, 从而引起一系列心理和生理疾病 ${ }^{[1]}$ 。减轻或 消除疲劳已成为现代科学研究的重要课题。已有 研究表明,高原环境中高寒、低压性缺氧、疲劳等 是对人体产生不良影响的主要因素, 影响高原工 作者体力和军事作业能力 ${ }^{[2-4]}$ 。因此, 积极研发具 有抗缺氧、抗疲劳的药物对于增强高原工作者心 理生理健康水平、提高军事作业能力具有十分重 要的意义 ${ }^{[5-6]}$ 。

白杨素 $(5,7$-二羟基黄酮 $)$ 是存在于大多数植 物中的天然黄酮化合物之一, 在蜂胶和蜂蜜中含 量较高 ${ }^{[7]}$, 具有抗氧化、抗抑郁、抗病毒、抗炎等广 泛的药理活性 ${ }^{[8-10]}$ 。白杨素的溶解性差, 生物利 用度低, 其临床药物开发受到很大的限制 ${ }^{[11]}$ 。生 物活性研究发现, 白杨素进行结构改造而得到的 7-HEC (图 1) 对低压性缺氧致脑组织损伤具有明 显的保护作用 ${ }^{[12]}$, 并且能够减轻低压性缺氧诱导 的认知功能损伤 ${ }^{[13]}$ 。本研究拟通过 7-HEC 干预低 压性缺氧大鼠模型, 测定相关抗疲劳、生化指标及 组织病理学变化, 初步阐明 7-HEC对低压性缺氧 大鼠运动性疲劳的保护作用, 为 7-HEC 药理作用 的充分挖掘提供理论依据。<smiles>O=c1cc(-c2ccccc2)oc2cc(O)cc(O)c12</smiles>

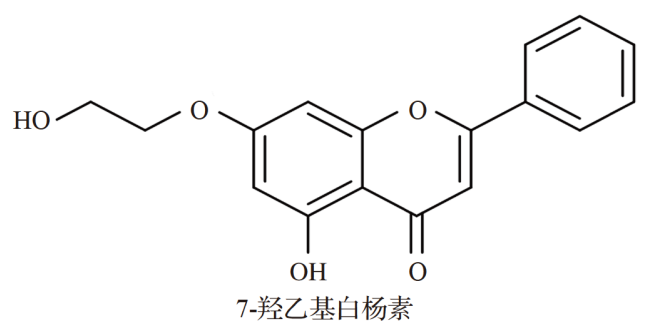

图 1 白杨素和7-羟乙基白杨素的化学结构式

Figure 1 The chemical structure of chrysin and 7hydroxyethyl chrysin

\section{1 材料与方法}

\section{1 试剂和仪器}

白杨素、BCA 法总蛋白定量试剂盒(A045-3)、 考马斯亮蓝法蛋白测试盒(A045-2)、血尿素氮测
试盒( 批号 20190525)、丙二醛测试盒 (A003-1)、 乳酸测试盒 (A019-2)、T-SOD 测试盒、ATP 酶测试 盒(A070-5)、糖原测定试剂盒 (A043) 为南京建成 生物工程研究所产品。7-HEC 由实验室自行 合成。

FLYDWC50-IIA 型低压性缺氧动物实验舱为 贵州风雷航空军械有限责任公司产品;SpectraMax i3 全自动苂光酶标仪为美国 Molecular Devices 公司 产品; 全自动样品快速研磨仪为上海净信实业发 展有限公司产品; AE240型分析天平为梅特勒-托 利多仪器 (上海) 有限公司产品; 全自动制冰机 (IMS-20) 为常熟市雪科电器有限公司产品; 浴旋 仪、低温高速离心机为德国 Sigma公司产品; 恒温 水浴箱为北京西城区医疗器械厂产品; NP80超微 量紫外分光光度计为德国 Implen公司产品。

\section{2 动物来源、分组及干预}

体重为180 220 g的无特定病原体 Wistar雄性 大鼠 40 只购自中国人民解放军联勤保障部队第九 四 $\bigcirc$ 医院动物实验科, 合格证号为 $\operatorname{SCXX}$ (军) 2012-0020。将40 只大鼠随机分为常氧对照组、缺 氧对照组、白杨素组和 7-HEC 组, 每组 10 只。分组 后, 各组每天灌胃给药 1 次, 连续 $3 \mathrm{~d}$, 其中常氧对 照组和缺氧对照组给予灭菌注射用水,白杨素组 和7-HEC组分别给予白杨素和7-HEC (350 mg/ $/ \mathrm{kg})$; 常氧对照组饲养于当地海拔, 其余三组均放人大 型低压性缺氧动物实验舱中, 以 $10 \mathrm{~m} / \mathrm{s}$ 速度减压 直至海拔 $7000 \mathrm{~m}$ 的压力, 缺氧处理 $3 \mathrm{~d}^{[14]}$ 。实验期 间大鼠自由进食、饮水, 定期更换垫料。实验过程 均严格按照 “3R” 原则进行, 遵守《关于善待实 验动物的指导性意见》, 实验废弃物及动物尸体 等按照《医疗废物管理条例》要求进行无害化 处理。

\section{3 力竭实验观察大鼠的运动耐受能力}

称重并记录各组大鼠体重,按大鼠体重的 $8 \%$ 负重, 在大鼠尾部缠绕相应质量的铅丝, 然后放人 水中进行力竭实验。每次实验放人 4 只大鼠, 同时 开始计时, 大鼠身体全部沉底并在 $3 \mathrm{~s}$ 内不上浮, 停 止计时, 统计游泳时间。常氧对照组在当地海拔 进行实验, 其余三组在低压性缺氧动物实验舱内 进行实验, 水温均为 $(25.0 \pm 0.5)^{\circ} \mathrm{C}$ 。

\section{4 艮酶法检测大鼠血尿素氮}

力竭实验结束即刻取大鼠全血标本于 $\mathrm{EP}$ 管 中, $2000 \times g$ 离心 $10 \mathrm{~min}$, 吸取上层血清分装后保存 
于 $-80{ }^{\circ} \mathrm{C}$ 冰箱待测。䐂酶法检测血尿素氮,按照血 尿素氮试剂盒说明书操作, 并计算血尿素氮浓度: 血尿素氮 $(\mathrm{mmol} / \mathrm{L})=[($ 样品吸光度值 - 空白吸光度 值) /(标准品吸光度值-空白吸光度值) $] \times$ 标准品 浓度 $(10 \mathrm{mmol} / \mathrm{L}) \times$ 样品稀释倍数。

$1.5 \mathrm{HE}$ 染色观察大鼠肝组织和骨骼肌组织病理 学改变

采血结束后, 每组随机选取 3 只大鼠, $10 \%$ 水 合氯醛麻醉, 立即取大鼠肝组织、骨骼肌组织标 本, 在 $10 \%$ 甲醛溶液中完全固定, 切片 (厚度为 2 3 mm), HE染色后在光镜下观察。

\section{6 比色法检测乳酸和肌、肝糖原含量}

采血结束后立即取大鼠肝组织、骨骼肌组织 标本, 准确称取各组织质量, 按质量 $(\mathrm{g})$ : 体积 $(\mathrm{mL})=1: 9$ 的比例加入等渗氯化钠溶液制成组织 匀浆, $1200 \times g 、 4^{\circ} \mathrm{C}$ 离心 $10 \mathrm{~min}$, 取上清液分装后保 存于 $-80^{\circ} \mathrm{C}$ 冰箱。采用比色法测定肝组织和骨骼 肌组织匀浆上清液中乳酸及肌肝糖原, 操作过程 严格按照说明书要求, 乳酸须在 $37^{\circ} \mathrm{C}$ 水浴反应 $10 \mathrm{~min}$ 后加人终止液, 检测吸光度值; 肌肝糖原须 在沸水中煮 5 min, 检测吸光度值。

1.7 差弪胺法和硫代巴比妥酸法测定 T-SOD 活性 和丙二醛含量

分别采用差弪胺法和硫代巴比妥酸法测定肝组 织和骨骼肌组织匀浆上清液中的 T-SOD 活性和丙 二醛含量, 具体操作均按照试剂盒说明书进行。

\section{8 统计学方法}

采用SPSS 19.0 软件进行统计分析。正态分 布的计量数据用均数 \pm 标准差 $(\bar{x} \pm s)$ 表示, 组间 比较采用单因素方差分析, $P<0.05$ 为差异有统计 学意义。

\section{2 结 果}

2.17 -HEC 对低压性缺氧大鼠负重游泳时间的 影响

与常氧对照组 $[(3.68 \pm 1.01) \mathrm{min}]$ 比较, 缺氧 对照组 [ (2.58 \pm 0.77$) \mathrm{min}]$ 游泳时间缩短 $(P<0.05)$; 与缺氧对照组比较, 白杨素组 $[(3.37 \pm$ $0.42) \mathrm{min}]$ 和 7-HEC 组 $[(4.04 \pm 1.30) \mathrm{min}]$ 游泳时 间延长 (均 $P<0.01$ ), 其中 7-HEC 组游泳时间较白 杨素组更长, 但差异无统计学意义 $(P>0.05)$ 。结 果提示, 低压性缺氧可使大鼠的运动能力下降, 白 杨素和 7-HEC 可提高下降的运动能力。
2.27 -HEC 对低压性缺氧大鼠血尿素氮生成速 率的影响

与常氧对照组 $\left[(1.63 \pm 0.44) \mathrm{mmol} \cdot \mathrm{L}^{-1} \cdot \mathrm{min}^{-1}\right]$ 比较, 缺氧对照组 $\left[(3.24 \pm 0.93) \mathrm{mmol} \cdot \mathrm{L}^{-1} \cdot \min ^{-1}\right]$ 血尿素氮生成速率明显加快 $(P<0.01)$; 与缺氧对照 组比较,白杨素组 $\left[(2.52 \pm 0.57) \mathrm{mmol} \cdot \mathrm{L}^{-1} \cdot \mathrm{min}^{-1}\right]$ 和 7-HEC 组 $\left[(2.22 \pm 0.60) \mathrm{mmol} \cdot \mathrm{L}^{-1} \cdot \mathrm{min}^{-1}\right]$ 血 尿素氮生成速率减小 $($ 均 $P<0.05)$, 其中 $7-H E C$ 组 血尿素氮生成速率较白杨素组更小 $(P<0.05)$ 。结 果提示, 白杨素、7-HEC可以增强低压性缺氧大鼠 的运动耐力, 且 7-HEC 作用效果更佳。

2.3 7-HEC 对低压性缺氧大鼠肝组织和骨骼肌 组织病理学变化的影响

常氧对照组肝组织结构完整,但细胞排列出 现疏松,结构基本保持稳定; 缺氧对照组细胞排列 散乱, 细胞间隙增大; 白杨素组情况有所好转; 7-HEC 组细胞形态基本恢复,结构趋于稳定。常 氧对照组大鼠骨骼肌结构完整, 肌纤维出现轻微 损伤, 纤维间隙增大, 骨骼肌出现断裂情况; 缺氧 对照组肌束界限开始模糊,间隙出现扩散现象, 有 大量炎症细胞浸润,肌纤维断裂明显; 白杨素组和 7-HEC 组肌纤维形态趋于正常,炎症细胞浸润减 少,肌纤维排列整齐,纤维间隙减小。见图 2 。结 果提示, 白杨素、7-HEC可以改善低压性缺氧对大 鼠肝组织和骨骼肌组织的损伤。

2.4 7-HEC 对低压性缺氧大鼠肌、肝糖原含量和 乳酸生成速率的影响

与常氧对照组比较, 缺氧对照组骨骼肌组织 和肝组织中糖原含量均减少, 尤以肝组织中糖原含 量减少显著; 乳酸生成速率均增加 (均 $P<0.05)$ 。 与缺氧对照组比较, 白杨素组和 7-HEC 组肝组织 中糖原含量均增加 (均 $P<0.05) ; 7-H E C$ 组肝组织 和骨骼肌组织中乳酸生成速率均减小 $($ 均 $P<$ $0.05)$ 。见表 1 。结果提示, 7-HEC 可以增加低压 性缺氧大鼠糖原含量,调节能量代谢。

2.57 -HEC 对低压性缺氧大鼠丙二醛生成速率 和T-SOD 活性的影响

与常氧对照组比较, 缺氧对照组骨骼肌组织 和肝组织中丙二醛生成速率均增加, T-SOD 活性 均降低 (均 $P<0.01$ ); 与缺氧对照组比较, 7-HEC 组 肝组织和骨骼肌组织中丙二醛生成速率均减小, T-SOD 活性均增加 $($ 均 $P<0.05)$ 。见表 2 。结果提 示, 7-HEC 可以保护低压性缺氧大鼠的机体损伤, 


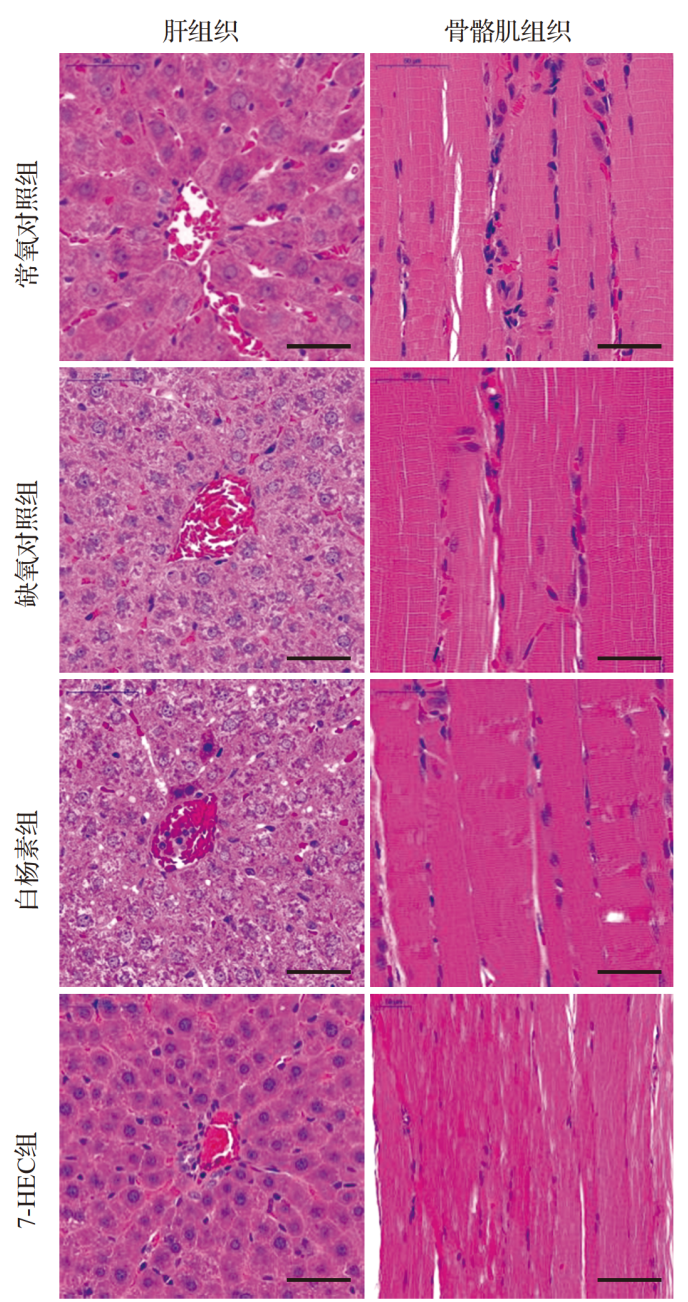

常氧对照组肝组织除细胞间隙增大外未见明显损伤; 缺氧对照组肝组织细胞排列散乱, 出现大量圆形空泡, 且炎 症细胞浸润明显; 白杨素组和7-HEC组肝组织细胞排列趋于 正常,炎症细胞浸润减少, 7-HEC组减少更明显. 常氧对照组 骨骼肌结构完整, 未见明显损伤; 缺氧对照组肌纤维明显断 裂、肿胀,炎症细胞浸润明显, 肌束界限模糊; 白杨素组和 7-HEC组肌纤维排列整齐, 炎症细胞显著减少, 骨骼肌细胞 趋于正常,且以7-HEC组更明显. 7-HEC:7-差乙基白杨素. 标尺 $=200 \mu \mathrm{m}$.

图 2 大鼠肝组织和骨骼肌组织病理学变化(HE染色)

Figure 2 Histopathological changes of liver and skeletal muscle tissues in rats (HE staining)
增强大鼠的抗氧化活性。

\section{3 讨 论}

我国高原地区战略地位极其重要,而其特殊 的低压性缺氧环境严重威胁当地军民的身心健 康。在缺氧情况下, 机体的活动能力明显下降, 极 易感到疲劳 ${ }^{[15]}$ 。本研究团队前期对白杨素进行 结构改造得到 7-HEC, 发现其对低压性缺氧致脑 组织损伤和认知功能损伤的保护作用更强 ${ }^{[12-13]}$ 。 本研究观察了 7-HEC 对低压性缺氧大鼠运动性疲 劳的影响。

力竭实验被广泛用于评估实验动物运动耐 受能力, 负重游泳时间可以反映大鼠抗疼劳能 力 ${ }^{[16]}$ 。本文资料表明, 7-HEC 可延长大鼠负重 游泳时间。运动性疲劳的主要表现有能量物质 消耗、疲劳物质积累以及运动过程中神经系统、 各种酶活性的改变 ${ }^{[17]}$ 。血尿素氮是评价机体运 动耐力的重要指标, 机体在缺氧条件下出现糖 代谢功能不足, 进一步引起蛋白质分解供能, 血 尿素氮含量升高 ${ }^{[18]}$ 。Zhu 等 ${ }^{[19]}$ 研究表明, 大鼠 运动疲劳后体内血尿素氮水平升高, 给予螺旋 藻多糖后可显著降低血尿素氮水平。本文资料 显示, 大鼠在低压性缺氧条件下进行力竭实验, 体内蛋白质急剧消耗, 血尿素氮生成速率增加, 而在给予 7-HEC后, 大鼠体内血尿素氮生成速 率明显减小, 提示 7-HEC 可以增强大鼠的运动 耐力。乳酸积累和糖原消耗是反映疲劳的主要 指标。乳酸在体内大量积累, 进一步破坏酸碱 平衡, 造成器官损伤, 导致疲劳 ${ }^{[20]}$; 机体运动过 程消耗的能量主要以糖原的形式存在于肝脏和 肌肉中 ${ }^{[21]}$, 肝组织和肌肉组织的结构完整是糖 异生和糖酵解的重要前提。在缺氧条件下, 肝 组织和骨骼肌组织的病理学变化明显, 肝糖原

表 1 各组糖原含量和乳酸生成速率比较

Table 1 Glycogen contents and lactate generation rate in each group

$(\bar{x} \pm s)$

\begin{tabular}{|c|c|c|c|c|c|}
\hline \multirow{2}{*}{ 组 别 } & \multirow{2}{*}{$n$} & \multicolumn{2}{|c|}{ 糖原含量 $(\mathrm{mg} / \mathrm{g})$} & \multicolumn{2}{|c|}{ 乳酸生成速率 $\left(\mathrm{mg} \cdot \mathrm{g}^{-1} \cdot \mathrm{min}^{-1}\right)$} \\
\hline & & 肝组织 & 骨骼肌组织 & 肝组织 & 骨骼肌组织 \\
\hline 常氧对照组 & 10 & $45.04 \pm 4.85$ & $1.81 \pm 0.42$ & $0.10 \pm 0.02$ & $0.19 \pm 0.06$ \\
\hline 缺氧对照组 & 10 & $15.16 \pm 2.69^{* *}$ & $1.46 \pm 0.49$ & $0.14 \pm 0.05^{*}$ & $0.26 \pm 0.06^{*}$ \\
\hline 白杨素组 & 10 & $20.27 \pm 4.18^{\#}$ & $1.43 \pm 0.40$ & $0.11 \pm 0.01$ & $0.17 \pm 0.06^{\#}$ \\
\hline 7-HEC组 & 10 & $18.89 \pm 2.88^{\#}$ & $1.78 \pm 0.48$ & $0.10 \pm 0.03^{\#}$ & $0.18 \pm 0.07^{\#}$ \\
\hline
\end{tabular}

与常氧对照组比较, ${ }^{*} P<0.05,{ }^{* *} P<0.01$; 与缺氧对照组比较, ${ }^{*} P<0.05$. 7-HEC: 7-差乙基白杨素. 
表 2 各组丙二醛生成速率和T-SOD活性比较

Table 2 MDA generation rate and T-SOD activity in each group

$(\bar{x} \pm s)$

\begin{tabular}{|c|c|c|c|c|c|}
\hline \multirow{2}{*}{ 组 别 } & \multirow{2}{*}{$n$} & \multicolumn{2}{|c|}{ 丙二醛生成速率 $\left(\mathrm{nmol} \cdot \mathrm{mg}^{-1} \cdot \mathrm{min}^{-1}\right)$} & \multicolumn{2}{|c|}{ T-SOD活性 $(\mathrm{U} / \mathrm{mL})$} \\
\hline & & 肝组织 & 骨骼肌组织 & 肝组织 & 骨骼肌组织 \\
\hline 常氧对照组 & 10 & $0.77 \pm 0.29$ & $0.48 \pm 0.15$ & $2.13 \pm 0.11$ & $6.53 \pm 0.54$ \\
\hline 缺氧对照组 & 10 & $1.37 \pm 0.48^{* *}$ & $0.87 \pm 0.19^{* *}$ & $1.87 \pm 0.01^{* *}$ & $5.46 \pm 0.42^{* *}$ \\
\hline 白杨素组 & 10 & $0.81 \pm 0.14^{\# \#}$ & $0.50 \pm 0.14^{\# \#}$ & $2.52 \pm 0.30^{\# \#}$ & $5.20 \pm 1.31$ \\
\hline 7-HEC组 & 10 & $0.78 \pm 0.28^{\# \#}$ & $0.63 \pm 0.11^{\# \#}$ & $2.68 \pm 0.12^{\# \#}$ & $6.52 \pm 0.96^{\#}$ \\
\hline
\end{tabular}

与常氧对照组比较, ${ }^{* *} P<0.01$; 与缺氧对照组比较, ${ }^{\#} P<0.05,{ }^{\# *} P<0.01$. 7-HEC:7-羟乙基白杨素; T-SOD: 总超氧化物歧化酶.

和肌糖原大量消耗; 给予 7-HEC 后, 肝组织和骨 骼肌组织的炎症细胞浸润减少, 病理学变化改 善, 乳酸生成减少, 肌肝糖原含量有所增加, 说 明 7-HEC 可能通过调节能量代谢对低压性缺氧 大鼠起到抗疲劳的作用。

疲劳的生理效应与氧化应激有关, 缺氧条件 下体内产生的大量活性氧攻击生物膜中的酶和不 饱和脂肪酸, 致使机体发生脂质过氧化, 产生丙二 醛等过氧化物, 对机体造成损伤 ${ }^{[22]}$ 。T-SOD 是自 由基清除重要的氧化酶, 可有效清除超氧阴离子, 预防机体氧化损伤。机体在缺氧条件下抗氧化酶 活性降低, 抗氧化系统失衡, 极易受到过氧化物攻 击, 从而出现损伤 ${ }^{[23-24]}$ 。本文资料显示, 7-HEC 可 提高 T-SOD 活性, 减少丙二醛生成, 恢复细胞功 能, 进而改善机体损伤, 达到缓解疲劳的作用。

综上所述, 7-HEC 对低压性缺氧大鼠运动性 疲劳具有保护作用, 其机制可能与调节能量代谢、 抑制脂质过氧化、增强抗氧化能力有关。

\section{利益冲突 所有作者均声明不存在利益冲突}

\section{参考文献}

[1] 谢红军, 罗布占堆, 阿呷尔希, 等. 芫参颗粒对小鼠运 动能力和抗疲劳作用研究 $[\mathrm{J}]$. 西藏医药, 2020, 41(4): 17-20.

XIE Hongjun, NorBu Zhandui, AGA ErBu, et al. Effect of yuanshen granules on exercise capacity and antifatigue in mice $[\mathrm{J}]$. Tibetan Medicine, 2020, 41 (4): 17-20. (in Chinese)

[2] LIU C, SHAO C, DU Q, et al. Mechanism and effects of fructose diphosphate on anti-hypoxia fatigue and learning memory ability $[\mathrm{J}]$. Can J Physiol Pharmacol, 2020, 98(10): 733-740.

[3] 李洋洋, 石 路, 张延猛, 等. 高原低氧运动人体能量 代谢变化及红景天的干预作用 $[\mathrm{J}]$. 西北国防医学杂 志, 2016, 37(6): 351-353.
LI Yangyang, SHI Lu, ZHANG Yanmeng, et al. Effects of Rhodiola rosea on human energy metabolism under physical load during chronic high altitude exposure $[\mathrm{J}]$. Medical Journal of National Defending Forces in Northwest China, 2016, 37 (6): 351-353. (in Chinese)

[4] 张 㑆, 陈景元, 李维国, 等. 高原高寒地区卫勤保障 实践与思考 $[\mathrm{J}]$. 西北国防医学杂志，2014，35(5): 497-499.

ZHANG Kan, CHEN Jingyuan, LI Weiguo, et al. Practice and thinking on health service support in plateau and alpine areas $[\mathrm{J}]$. Medical Journal of National Defending Forces in Northwest China, 2014, 35(5): 497-499. (in Chinese)

[5] WU R, SUN Y, ZHOU T, et al. Arctigenin enhances swimming endurance of sedentary rats partially by regulation of antioxidant pathways $[\mathrm{J}]$. Acta Pharmacol Sin, 2014, 35(10): 1274-1284.

[6] 李茂星, 王先敏, 毛 婷, 等. 㭛子黄色素对模拟高原 缺氧小鼠运动性疲劳的改善作用 $[\mathrm{J}]$. 解放军药学学 报, 2017, 33(1): 28-32.

LI Maoxing, WANG Xianmin, MAO Ting, et al. Alleviation of exercise-induced fatigue by Gardenia Yellow Pigment at high altitude $[\mathrm{J}]$. Pharmaceutical Journal of Chinese People's Liberation Army, 2017, 33(1): 28-32. (in Chinese)

[7] BALAM F H, AHMADI Z S, GHORBANI A. Inhibitory effect of chrysin on estrogen biosynthesis by suppression of enzyme aromatase (CYP19): a systematic review[J/OL]. Heliyon, 2020, 6(3): e03557.

[8] CUETO-ESCOBEDO J, ANDRADE-SOTO J, LIMAMAXIMINO M, et al. Involvement of GABAergic system in the antidepressant-like effects of chrysin $(5,7-$ dihydroxyflavone) in ovariectomized rats in the forced swim test: comparison with neurosteroids $[\mathrm{J}]$. Behav Brain Res, 2020, 386: 112590.

[9] WANG J, ZHANG T, DU J, et al. Anti-enterovirus 71 effects of chrysin and its phosphate ester[J/OL]. PLoS One, 2014, 9(3): e89668.

[10] HWANG S H, KIM H Y, ZUO G, et al. Anti-glycation, carbonyl trapping and anti-inflammatory activities of chrysin derivatives[J]. Molecules, 2018, 23(7): 1752. 
[11] MANI R, NATESAN V. Chrysin: sources, beneficial pharmacological activities, and molecular mechanism of action[J]. Phytochemistry, 2018, 145: 187-196.

[12] 景临林, 杨 颖, 邵 瑾, 等, 7-差乙基白杨素对低 压低氧致脑组织损伤的保护作用 $[\mathrm{J}]$. 中国现代应 用药学, 2020, 37(9): 1025-1029.

JING Linlin, YANG Ying, SHAO Jin, et al. Protective effect of 7-HEC on brain tissue damage induced by hypobaric hypoxia[J]. Chinese Journal of Modern Applied Pharmacy, 2020, 37(9): 1025-1029. (in Chinese)

[13］景临林, 杨 颖, 邵 瑾, 等, 7-羟乙基白杨素对低 压低氧大鼠认知功能的保护作用及机制研究 $[\mathrm{J}]$. 中国医院药学杂志, 2020, 40(15): 1622-1626.

JING Linlin, YANG Ying, SHAO Jin, et al. Protective effects and mechanism of 7-HEC against hypobaric hypoxia-induced cognitive impairments in $\operatorname{rats}[\mathrm{J}]$. Chinese Journal of Hospital Pharmacy, 2020, 40(15):1622-1626. (in Chinese)

[14] 陶文迪, 田秀玉, 李茂星, 等. 黄芪水提取物对高原缺 氧大鼠运动能力的影响 $[\mathrm{J}]$. 解放军医药杂志, 2019, 31(12): 12-18.

TAO Wendi, TIAN Xiuyu, LI Maoxing, et al. Effect of astragalus membranaceus aqueous extract on ability of plateau hypoxia exercise in rats $[\mathrm{J}]$. Medical \& Pharmaceutical Journal of Chinese People's Liberation Army, 2019, 31 (12): 12-18. (in Chinese)

[15] ZHOU S, WANG Y, TIAN H, et al. Anti-fatigue effects of Panax notoginseng in simulation plateau-condition mice[J]. Pharmacogn Mag, 2012, 8(31): 197-201.

[16] WANG S Y, HUANG W C, LIU C C, et al. Pumpkin (Cucurbita moschata) fruit extract improves physical fatigue and exercise performance in $\operatorname{mice}[\mathrm{J}]$. Molecules, 2012, 17(10): 11864-11876.
[17] REN G, YI S, ZHANG H, et al. Ingestion of soy-whey blended protein augments sports performance and ameliorates exercise-induced fatigue in a rat exercise model[J]. Food Funct, 2017, 8(2): 670-679.

[18] SHIN I S, KIM D H, JANG E Y et al. Anti-fatigue properties of cultivated wild ginseng distilled extract and its active component panaxydol in rats $[\mathbf{J}]$. J Pharmacopuncture, 2019, 22(2): 68-74.

[19] ZHU M, ZHU H, DING X, et al. Analysis of the antifatigue activity of polysaccharides from Spirulina platensis: role of central 5-hydroxytryptamine mechanisms $[J]$. Food Funct, 2020, 11(2): 1826-1834.

[20] ZHAO H P, ZHANG Y, LIU Z, et al. Acute toxicity and anti-fatigue activity of polysaccharide-rich extract from corn silk $[\mathrm{J}]$. Biomed Pharmacother, 2017, 90: 686-693.

[21] CHEN Y B, WANG Y F, HOU W, et al. Effect of Bcomplex vitamins on the antifatigue activity and bioavailability of ginsenoside Re after oral administration[J]. J Ginseng Res, 2016, 41(2): 209-214.

[22] XI C, LIANG X, CHEN C, et al. Hypoxia induces internalization of $\kappa$-opioid receptor $[\mathrm{J}]$. Anesthesiology, 2017, 126(5): 842-854.

[23 ] SAKR H F, ABBAS A M, EI Samanoudy A Z. Effect of vitamin $\mathrm{E}$ on cerebral cortical oxidative stress brainderived neurotrophic factor gene expression induced by hypoxia and exercise in rats $[\mathrm{J}]$. J Physiol Pharmacol, 2015, 66(2): 191-202.

[24] CHI A, LI H, KANG C, et al. Anti-fatigue activity of a novel polysaccharide conjugates from Ziyang green tea $[J]$. Int J Biol Macromol, 2015, 80: 566-572.

$$
\text { [本文编辑 沈 敏 余 方] }
$$

\section{吕志民教授当选欧洲科学院外籍院士}

浙江大学转化医学研究院吕志民教授当选2021年新增的欧洲科学院外籍院士。欧洲科学院 (Academia Europaea)成 立于1988年,总部设在英国伦敦,是由欧洲多国科学部长共同倡导创立、由英国皇家学会等多个代表欧洲国家最高学术 水平的国立科学院共同发起成立的国际科学组织。该院院士主要来自欧洲国家,目前共有院士约 4500 名,其中包括 74 名 诺贝尔奖获得者、7名图灵奖获得者、15名菲尔兹奖获得者。 